\title{
Efficacy and Safety of Glecaprevir/Pibrentasvir in Patients with Chronic HCV Infection
}

\author{
Xiaoqing Liu and Peng $\mathrm{Hu}^{*}$ (C) \\ Department of Infectious Diseases, Institute for Viral Hepatitis, The Key Laboratory of Molecular Biology for Infectious Dis- \\ eases, Chinese Ministry of Education, The Second Affiliated Hospital of Chongqing Medical University, Chongqing, China
}

\begin{abstract}
Hepatitis C virus (HCV) infection is a major cause of endstage liver disease, including decompensated cirrhosis and hepatocellular carcinoma. Over $95 \%$ of patients with $\mathrm{HCV}$ infection have achieved sustained virologic response at 12 weeks under the treatment of several pan-genotypic regimens approved for patients with HCV infection. The glecaprevir/pibrentasvir (G/P) regimen has some features that distinguish it from others and is the only 8-week regimen approved for treatment-naive patients and patients experienced in regimens containing (peg)interferon, ribavirin, and/ or sofosbuvir, without an HCV NS3/4A protease inhibitor or NS5A inhibitor (except those with genotype 3 ). This review aims to summarize the efficacy and safety of G/P in HCVinfected patients from clinic trials and real-world studies, including those who have historically been considered difficult to cure.
\end{abstract}

Citation of this article: Liu X, Hu P. Efficacy and safety of glecaprevir/pibrentasvir in patients with chronic HCV infection. J Clin Transl Hepatol 2021;000(000):000-000. doi: 10.14218/JCTH.2020.00078.

\section{Introduction}

Hepatitis C virus (HCV) infection threatens the health of people around the world. According to the World Health Organization, about 71 million people worldwide suffer from chronic HCV infection, and in 2016 approximately 399,000 patients died from end-stage HCV infection, mainly from cirrhosis and hepatocellular carcinoma. ${ }^{1}$ Direct-acting antiviral agent (DAA)-induced sustained virologic response (SVR) has been associated with a reduction in the risk of cirrhosis, hepatocellular carcinoma, and mortality. ${ }^{2,3}$

All of the three pan-genotypic antiviral regimens [sofos-

Keywords: Hepatitis C; Mavyret; Glecaprevir; Pibrentasvir; Treatment outcome. Abbreviations: $A E$, adverse event; $A R A$, acid-reducing agent; $C K D$, chronic kidney disease; DAA, direct-acting antiviral agent; DDI, drug-drug interaction; $G / P$ glecaprevir/pibrentasvir; HCV, hepatitis C virus; ITT, intention to treat; OATP, organic anion transporting polypeptide; OST, opioid substitution therapy; PI, protease inhibitor; PPI, proton pump inhibitor; PRS, regimens containing (peg) interferon, ribavirin, and/or sofosbuvir, without an HCV NS3/4A PI or NS5A inhibitor; SVR, sustained virologic response; SVR12, SVR at 12-week post-treatment. Received: 18 August 2020; Revised: 03 December 2020; Accepted: 22 December 2020

*Correspondence to: Peng Hu, Department of Infectious Diseases, Institute for Viral Hepatitis, The Key Laboratory of Molecular Biology for Infectious Diseases, Chinese Ministry of Education, The Second Affiliated Hospital of Chongqing Medical University, 74 Linjiang Road, Yuzhong District, Chongqing 400010 China. Tel: +86-23-62887083, Fax: +86-23-63703790, E-mail: hp_cq@163. com, hupengcq@hospital.cqmu.edu.cn buvir/velpatasvir, sofosbuvir/velpatasvir/voxilaprevir, glecaprevir/pibrentasvir (G/P)] approved for the treatment of patients with chronic $\mathrm{HCV}$ infection in recent years have high efficacy, and more than $95 \%$ of patients administered them have achieved SVR at 12-week post-treatment (SVR12). ${ }^{3-7}$

However, the fixed-dose combination of Glecaprevir (300 $\mathrm{mg}$ ) and pibrentasvir (120 mg), an all-oral, once-daily, ribavirin-free pan-genotypic regimen approved in 2017, has certain characteristics that distinguish it from other pangenotypic antiviral regimens, including shorter duration of therapy for most patients; it has been approved for use in patients with end-stage renal disease and adolescent patients aged $12-17$ years. ${ }^{8}$

Both glecaprevir and pibrentasvir have potent anti-HCV activity across genotypes 1 through 6 in vitro, which harbor a high genetic barrier to resistance. 8 The former is a second-generation NS3/4A protease inhibitor (PI), essential for the cleavage of the HCV unprocessed polyprotein and viral replication; the latter is a second-generation NS5A inhibitor, critical for viral RNA replication and virion assembly. ${ }^{9}$

Glecaprevir and pibrentasvir are mainly excreted through the biliary-fecal route, with only a minor fraction (less than $1 \%$ ) excreted in urine; renal impairment and hemodialysis appeared to have no significant influence on glecaprevir or pibrentasvir exposures. ${ }^{8,10}$ Compared with patients with normal renal function, HCV-infected patients with end-stage renal disease, including dialysis, were observed to have an $86 \%$ increase in glecaprevir and a $54 \%$ increase in area under the curve for pibrentasvir. ${ }^{8}$ In chronic HCV-infected patients with compensated cirrhosis, exposure to glecaprevir was $160 \%$ higher and pibrentasvir exposures showed little difference in outcome compared to patients without cirrhosis. ${ }^{10}$ Meanwhile, there was no statistically significant difference in the exposure rates of gelcaprevir and pibrentasvir between Japanese or Han Chinese and Whites. ${ }^{11}$ But, we should note that patients with decompensated cirrhosis are not recommended for treatment with a PI-containing regimen (e.g., glecaprevir, grazoprevir, and voxilaprevir).

Most patients with chronic HCV infection are at risk of drug-drug interactions (DDIs) with co-medication. ${ }^{12}$ Both glecaprevir and pibrentasvir are substrates and inhibitors of P-glycoprotein and breast cancer resistance protein. Moreover, glecaprevir is a substrate and inhibitor for organic anion transporting polypeptide (OATP) $1 \mathrm{~B} 1$ and OATP1B3. Pibrentasvir is an inhibitor of OATP1B1/3. Therefore, coadministration of G/P with drugs that inhibit hepatic P-glycoprotein, breast cancer resistance protein, or OATP1B1/3 may increase the plasma concentrations of glecaprevir and/or pibrentasvir. ${ }^{8}$ As per the drug label, G/P is contraindicated in combination with atazanavir or rifampin. ${ }^{8}$

The main purpose of this review is to comprehensively evaluate the efficacy and safety of G/P in patients with HCV infection, including those in the so-called "special population" that have historically been considered difficult to treat. 
Liu X. et al: G/P effectiveness and tolerability

Efficacy

The clinic trails and real-world studies that have evaluated the efficacy of G/P against HCV are summarized in Tables 1 and $2,{ }^{13-47}$ respectively.

\section{Treatment-naïve or patients experienced in regimens containing (peg)interferon, ribavirin, and/or sofos- buvir, without an HCV NS3/4A PI or NS5A inhibitor, without cirrhosis}

In a pooled analysis of nine clinical trials, treatment-naïve or patients experienced in regimens containing (peg)interferon, ribavirin, and/or sofosbuvir, without an HCV NS3/4A PI or NS5A inhibitor (PRS) with HCV genotypes 1-6 infections but without cirrhosis achieved an overall SVR12 rate of $98 \%(943 / 965)$ in the intention-to-treat (ITT) population when treated for 8 weeks, which showed no significant difference from patients treated with 12 weeks $(1,060 / 1,076$, $99 \%) .{ }^{48}$ But, all 478 patients infected with genotype 3 were treatment-naïve in that study. CRETIN-1 and CRETAIN-2 were not included in the pooled analysis described previously, and the authors also demonstrated that G/P treatment for 8 weeks was highly effective in patients with HCV genotypes 1-2 infections without cirrhosis, of which $99.2 \%(128 / 129)$ and $98 \%(88 / 90)$ patients achieved SVR12, respectively. ${ }^{13,14}$ The ENDURANCE-5, 6 trial, an open-label, multicenter, phase $3 \mathrm{~b}$ trial, demonstrated that $98.6 \%(74 / 75)$ of non-cirrhotic patients infected with HCV genotype 5 or 6 treated with G/P for 8 weeks achieved SVR12. ${ }^{15}$

Data from a combined analysis of 18 real-world studies showed that the SVR rate for treatment-naïve patients without cirrhosis who received G/P treatment for 8 weeks was $98.2 \%(n=697)$ in the ITT population and $99.3 \%(n=3,657)$ in the modified ITT population. ${ }^{49}$ For patients with treatment experience, the SVR rate was $90.7 \%$ (49/54), among which 4 patients were lost to follow-up. ${ }^{16}$

\section{Treatment-naïve or PRS-experienced patients with compensated cirrhosis}

The efficacy of 8-week G/P reported in the EXPEDITION-8 clinical trial is comparable to the 12 -week regimen for treatment-naïve patients with genotypes 1-6 infections and compensated cirrhosis. ${ }^{17}$ The SVR rate was $97.7 \%(335 / 343)$ in the ITT population and $99.7 \%(334 / 335)$ in the per protocol population after treatment with G/P once daily for 8 weeks. ${ }^{17}$ After 12 weeks of treatment with $G / P$, the SVR12 rates of HCV genotype 3- and non-genotype 3-infected compensated cirrhosis patients without treatment experience were $97.1 \%$ $(67 / 69)^{50}$ and $100 \%(110 / 110), 18$ respectively.

For PRS-experienced patients with compensated cirrhosis and genotype non-3 infection, the EXPEDITION-1 trial reported that $97.2 \%(35 / 36)$ of patients treated for 12 weeks achieved SVR12, with one genotype 1a-infected patient relapsing at post-treatment week 8. ${ }^{18}$ The CRETAIN-1, subgroup 2 trial evaluated the efficacy of a 12-week course of G/P in 38 genotype 1-infected patients with compensated cirrhosis. All of the 38 patients $(100 \%)$ achieved SVR12, of which 12 were interferon-experienced. 13

Data from real-world studies showed that the SVR12 rate for patients with compensated cirrhosis who received G/P treatment was $97.8 \%(n=676)$ in the ITT population and $98.2 \%(\mathrm{~N}=822)$ in the modified ITT population. ${ }^{49}$ For treatment-naive patients with compensated cirrhosis treated for 12 weeks, the SVR12 rate was $99.0 \%(n=362) .49$ To our knowledge, currently there is no real-world data to evalu- ate the efficacy of G/P treatment for 8 weeks in treatmentnaïve patients with compensated cirrhosis.

\section{"Special population"}

\section{Patients with HCV genotype 3 infection}

Patients with HCV genotype 3 infection are among the most difficult to treat in the DAA-era. According to the studies described above, treatment-naïve and genotype 3-infected patients treated with G/P for 8 weeks had high SVR12 rate, regardless of cirrhosis, but the efficacy in PRS-experienced patients is still uncertain.

In the SURVEYOR-II trial, parts 1 and 2, the efficacy of $G / P$ for 12 weeks was studied in PR-experienced patients with genotype 3 infection and without cirrhosis. In total, $91.7 \%(22 / 24)$ achieved SVR12, with 1 patient having a breakthrough at treatment week 6 and 1 other relapsing at post-treatment week 8. ${ }^{19}$ The SURVEYOR-II trial, part 3, a partially-randomized, open-label, phase 3 study, assessed the efficacy of G/P in patients with genotype 3 infection with prior treatment experience, and found that $91 \%(20 / 22)$ and $96 \%(21 / 22)$ of PRS-experienced patients without cirrhosis achieved SVR12 for 12 weeks and 16 weeks, respectively. Among the 47 treatment-experienced patients with compensated cirrhosis, SVR12 were achieved by $96 \%$ (45/47) of patients treated with G/P for 16 weeks. ${ }^{20}$ An integrated cirrhosis of five phase 2 or 3 trials that evaluated $\mathrm{G} / \mathrm{P}$ in patients with chronic HCV genotype 3 infection also demonstrated G/P was efficacious for those patients, regardless of cirrhosis or prior treatment experience. ${ }^{50}$

Data from real-world studies also demonstrated that over $95 \%$ of HCV genotype 3-infected patients treated with the G/P regimen achieved SVR. ${ }^{21,49}$ But, we should point out that almost all of the above-mentioned research studies were conducted in areas where subtype $3 a$ is dominant, and subtype $3 \mathrm{~b}$ accounts for less than $1 \%$ of all genotype 3 cases. Therefore, more attention should be paid to the efficacy of G/P in HCV subtype 3b-infected patients. Nozaki et al. ${ }^{22}$ recently reported that only $33.3 \%(2 / 6)$ of patients with HCV subtype 3b infection achieved SVR12. Another study conducted by Tamori et al. ${ }^{23}$ found that only $50 \%(2 / 4)$ of patients with HCV genotype 3b infection achieved SVR12. Moreover, one out of three patients with genotype $3 a / b$ experienced virologic failure in the study of Toyoda et al. ${ }^{24}$

\section{Patients with severe renal impairment}

The investigators of the EXPEDITION-4 study examined the efficacy of G/P administered for 12 weeks in adults with chronic HCV genotypes 1-6 infections and stage 4 or 5 chronic kidney disease (CKD). ${ }^{25}$ Of the 104 patients enrolled, $44(42 \%)$ had prior treatment experience for $\mathrm{HCV}$, but treatment-experienced patients who had genotype 3 infection were excluded and $20(19 \%)$ had compensated cirrhosis at baseline. Up to $98 \%(102 / 104)$ of the patients achieved SVR12, with no patients experiencing virologic failure. ${ }^{25}$ In the phase 3 EXPEDITION-5 trial, the rate of SVR was $97 \%(98 / 101)$ in the ITT population and $100 \%(98 / 98)$ in the modified ITT population after treatment with G/P. Of the 101 enrolled adults with CKD stages $3 \mathrm{~b}, 4$ or 5,84 patients without cirrhosis were assigned to receive G/P for 8 weeks and 4 PRS-experienced patients with genotype 3 infection were assigned to 16 weeks of treatment; all others were in the 12-week treatment group. ${ }^{26}$ Real-world studies also demonstrated G/P was highly effective for patients with CKD stages 4-5 (including patients undergoing hemodialy- 


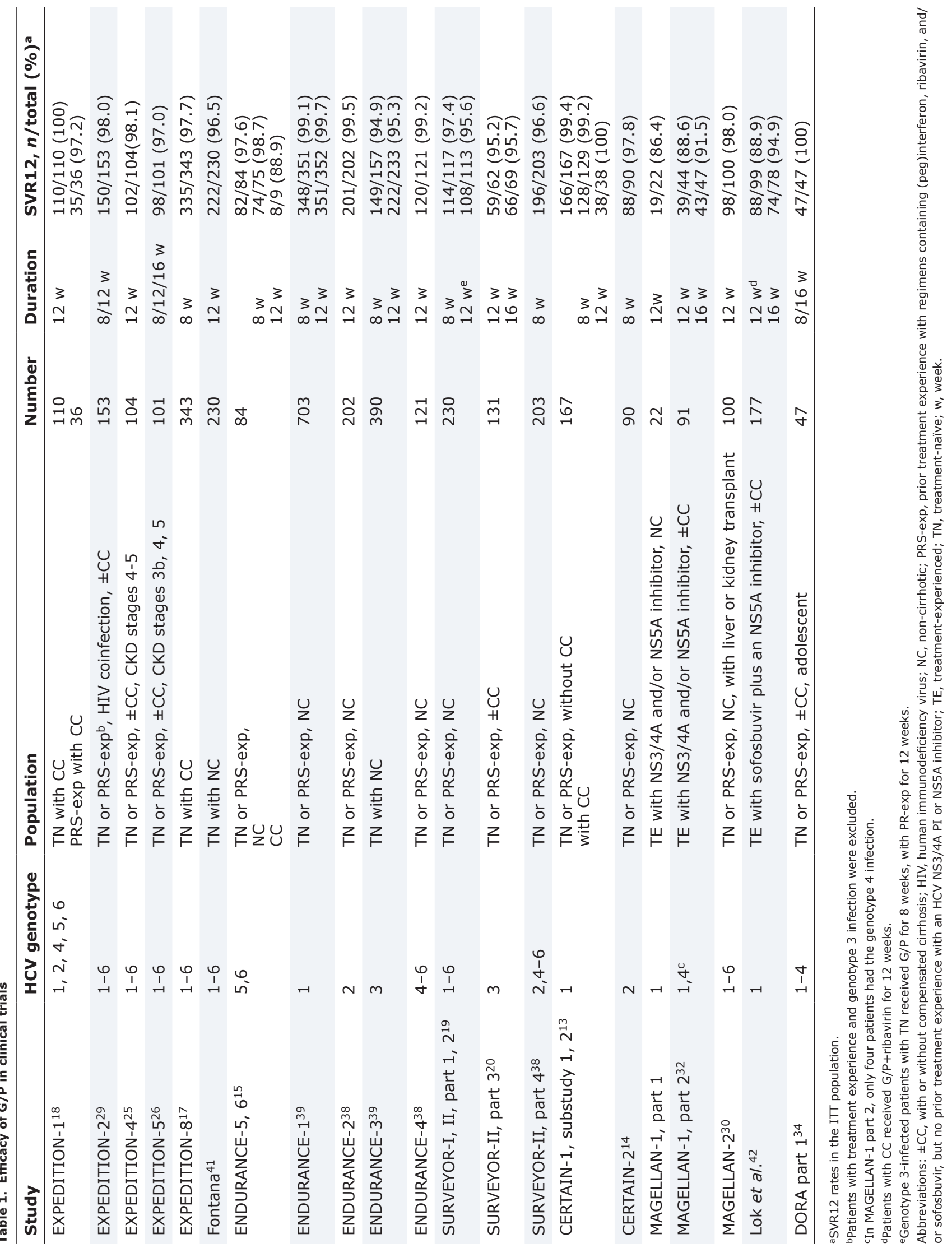




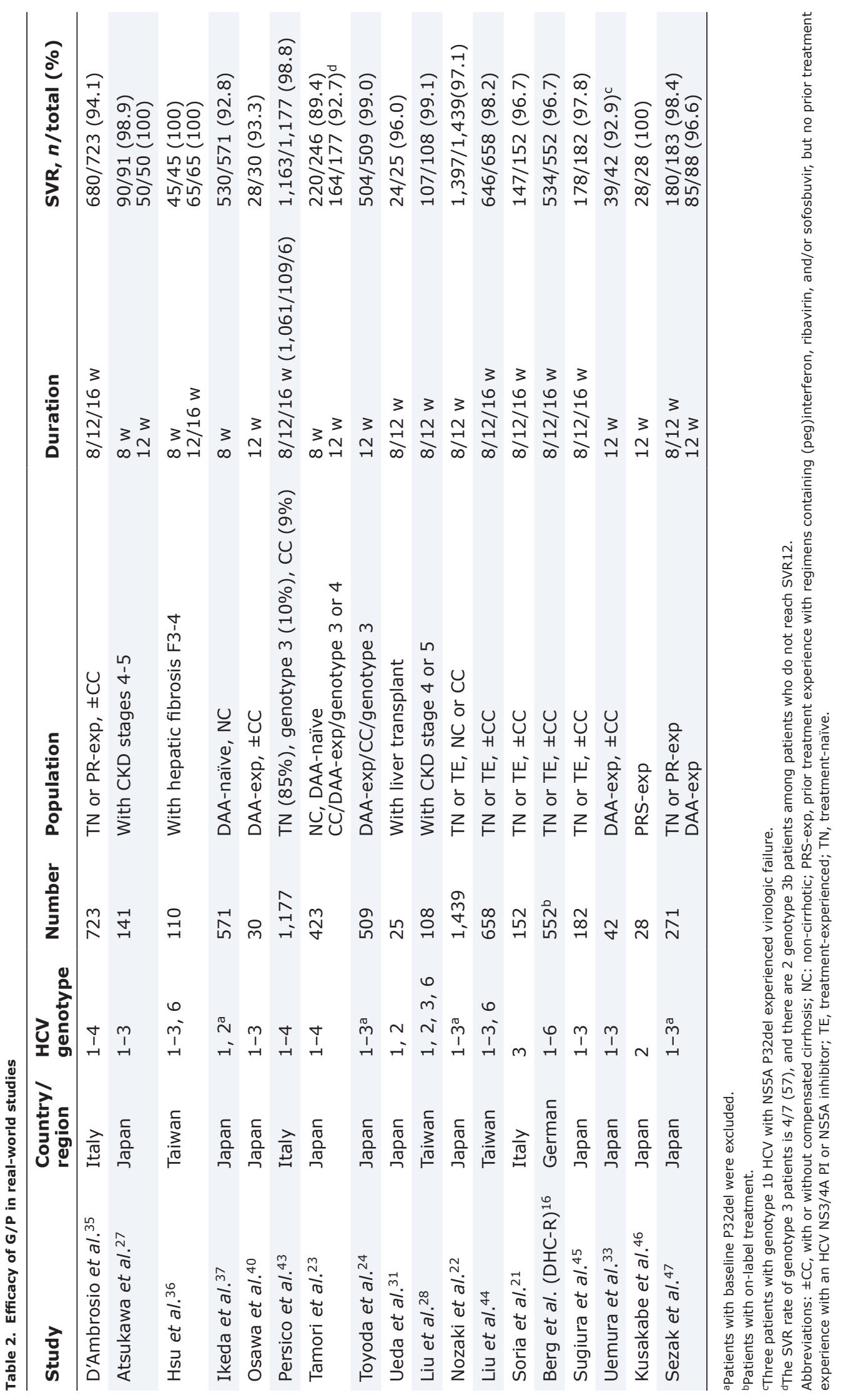


Liu X. et al: G/P effectiveness and tolerability

sis), and more than $98 \%$ of patients achieved SVR. $22,27,28,51$

\section{Patients with human immunodeficiency virus-1/HCV coinfection}

EXPEDITION-2 was a phase 3, multicenter study to evaluate the efficacy of G/P in 156 human immunodeficiency virus/ HCV-coinfected adults with compensated liver diseases.29 All patients with genotype 3 infection were treatment-naïve for HCV. Patients were either antiretroviral therapy-naïve or on a qualifying antiretroviral therapy regimen for at least 8 weeks. The antiretroviral therapy drugs used by more than 20 patients included tenofovir disoproxil fumarate, abacavir, emtricitabine, lamivudine, raltegravir, dolutegravir, and rilpivirine. As high as $99.3 \%(136 / 137)$ of the patients without cirrhosis treated for 8 weeks achieved SVR12, and $87.6 \%$ $(14 / 16)$ of the patients with compensated cirrhosis treated for 12 weeks achieved SVR12. No one experienced virologic failure, except one HCV treatment-naïve patient with genotype 3 infection and compensated cirrhosis. ${ }^{29}$

\section{Patients with liver or kidney transplantation}

MAGELLAN-2, a phase 3, open-label trial, evaluated the efficacy of G/P for 12 weeks in patients who had chronic HCV genotypes1-6 infections and had received a liver $(n=80)$ or kidney $(n=20)$ transplant at least 3 months prior. ${ }^{30}$ Patients with cirrhosis were excluded and all genotype 3-infected patients were treatment-naïve. Overall, SVR was achieved in $98 \%(98 / 100)$ of the patients, with one genotype 3ainfected patient who had received a liver transplant experiencing virologic relapse. ${ }^{30}$ Ueda et al. ${ }^{31}$ reported that $96 \%$ $(24 / 25)$ of patients with recurrent HCV infection after liver transplantation treated with 8 or 12 weeks of G/P achieved SVR12, including patients with severe renal impairment, liver cirrhosis, prior DAA experience, or jaundice after liver transplantation. But, no virologic failure occurred.

\section{Patients with psychiatric disorders}

An integrated analysis of 10 phase 2 and phase 3 clinical trials assessed the efficacy of G/P for 8,12 or 16 weeks in chronic HCV genotypes 1-6-infected patients with psychiatric disorders. 52 The overall patients' treatment adherence was very high $(>95 \%)$, regardless of whether there was a psychiatric disorder. Of the 2,522 patients receiving $\mathrm{G} / \mathrm{P}, 97.3 \%$ (768/789) of those with a psychiatric disorder achieved SVR12 compared to $97.5 \%(1,689 / 1,733)$ of those without a psychiatric disorder. Among all patients receiving a neuropsychiatric co-medication with potential DDIs with $G / P$, the SVR rate was still high, including quetiapine $(100 \%, 47 / 47)$, oxycodone $(93.8 \%, 76 / 81)$ and hydrocodone $(97.4 \%, 75 / 77) .52$ Meanwhile, patients with psychiatric disorder had overall high SVR rate $(98.9 \%)$ in the modified ITT population in real-word studies. 49

\section{Patients with NS3/4 PI and/or NS5A inhibitor treat- ment experience}

In part 1 of the MAGELLAN-1 trail, 22 genotype 1 -infected non-cirrhotic patients with past failure to NS3/4 PI and/ or NS5A inhibitor were treated with G/P for 12 weeks. ${ }^{53}$ SVR was achieved by $86 \%(19 / 22)$ in the ITT population and $95 \%(19 / 20)$ in the modified ITT population. Virologic failure occurred in one patient with past treatment expe- rience with both NS3/4A PI and NS5A inhibitor. ${ }^{53}$ MAGELLAN-1, part 2, a randomized, open-label, multicenter phase 3 study, enrolled 91 HCV genotypes 1 or 4-infected patients with compensated liver disease (with or without cirrhosis) who had past treatment experience with NS3/4A PI and/ or NS5A inhibitor. ${ }^{32}$ Patients enrolled were randomized to receive 12 or 16 weeks of G/P. Patients with NS3/4A PI experience alone (NS5A inhibitor-naïve) had 100\% SVR12, regardless of treatment duration. Patients with NS5A inhibitor experience alone (NS3/4A PI-naïve) had 88\% (14/16) and $94 \%(17 / 18)$ SVR rate among those treated with G/P for 12 weeks and 16 weeks, respectively. Patients with both NS3/4A PI and NS5A inhibitor experience had 79\% (11/14) and $81 \%(13 / 16)$ SVR rate among those treated for 12 weeks and 16 weeks, respectively. All (4/4) patients with genotype 4 infection achieved SVR12 and 10.3\% (9/87) of patients with chronic HCV genotype 1 infection experienced virologic failure. In CRETAIN-1 and -2 trials, The SVR rate in the HCV subtype1b-infected patients with PI and NS5A inhibitor experience was $93.3 \%(28 / 30)$. Virologic failure occurred in two patients with NS5A P32del.54

Research conducted by Uemura et al., ${ }^{33}$ in which 34 of 42 patients had both NS3 and NS5A inhibitors treatment experience, reported that the SVR12 rate of chronic hepatitis c patients with prior DAA treatment experience treated with G/P for 12 weeks was $92.9 \%$ (39/42). All genotype $1 \mathrm{~b}$-infected patients carrying the NS5A P32del (3/35) experienced virologic failure. ${ }^{33}$

\section{Adolescent patients aged 12-17}

The DORA part 1, nonrandomized, open-label, multicenter clinical trial assessed the efficacy of G/P in adolescent patients with chronic HCV genotypes 1-4 infections. ${ }^{34}$ Of the 47 enrolled patients, 36 patients were HCV treatment-naïve and the others were interferon-based regimen-experienced. Except for three patients with HCV genotype 3 infections and treatment experience, who received G/P for 16 weeks, all patients received 8 weeks of therapy. The SVR12 rate of overall patients was $100 \%(47 / 47){ }^{34}$

\section{Patients who used drugs recently or were receiving opioid substitution therapy}

Among 15.6 million patients who inject drugs, an estimated $52.3 \%$ are $\mathrm{HCV}$-antibody positive. ${ }^{55} \mathrm{~A}$ pooled analysis of clinic trials revealed that SVR12 was achieved by $92.9 \%$ $(91 / 98), 97.0 \%(592 / 610)$, and $99.5 \%(1,106 / 1,111)$ of recent, former, and non-drug users, respectively. But, the overall rates of virologic failure were $\leq 1.5 \%$, regardless of drug use status. ${ }^{56}$ An integrated analysis of eight clinical trials compared the efficacy of G/P in HCV-infected patients receiving opioid substitution therapy (OST) and those not receiving OST. SVR rates were $96.2 \%(151 / 157)$ and $97.9 \%$ $(2,055 / 2,099)$ in the OST and non-OST patients, respectively. ${ }^{57}$ Methadone was the most commonly prescribed OST (76\%). ${ }^{57}$ An integrated analysis of 18 real-word studies demonstrated that $98.9 \%$ of chronic HCV patients using OST achieved SVR12 in the modified ITT analysis. ${ }^{49}$

\section{Patients with concurrent use of acid-reducing agents}

Proton pump inhibitors (PPIs) were among the most frequently used co-medication in patients treated for chronic HCV infection. ${ }^{12}$ An integrated analysis of nine phase 2 and phase 3 clinic trails showed that in patients treated with $G / P$, SVR rates were $97.0 \%(389 / 401)$ and $97.5 \%(1,918 / 1,968)$ 
Liu X. et al: G/P effectiveness and tolerability

for patients using concomitant acid-reducing agents (ARAs) and those not concurrently using ARAs, respectively. ${ }^{58}$ SVR was achieved in $96.3 \%(105 / 109)$ of patients taking a highdose PPI and $97.4 \%(150 / 154)$ taking a low-dose PPI. The overall virologic failure rate in patients taking an ARA was less than $1 \%(4 / 401)$ compared with $1.5 \%(29 / 1,968)$ in patients not taking any ARAs. ${ }^{58}$ The SVR rate of patients with chronic HCV infection receiving PPI was $97.9 \%(n=180)$ in real-world studies. ${ }^{49}$

\section{Tolerability and safety}

HCV N3/4A PIs have been shown to have concentration-dependent hepatotoxicity and are contraindicated in patients with decompensated cirrhosis. Therefore, patients with factors known to affect the exposure to glecaprevir need to be carried out, including those on patients with renal impairment, DDIs, advanced age, female sex, and cirrhosis status. ${ }^{59}$

A pooled analysis of nine clinical trials evaluated the tolerability and safety of $\mathrm{G} / \mathrm{P}$ in chronic HCV-infected patients and studied by subgroup. ${ }^{60}$ In general, the prevalence of adverse events (AEs) were $68 \%(1,603 / 2,369)$, and the most commonly reported AEs with an incidence rate exceeding $5 \%$ included headache, fatigue, nausea, and pruritus. ${ }^{60}$ Serious AEs occurred in $73(3 \%)$ patients but only one $(<1 \%)$ was reported as DAA-related. Although $55 \%(11 / 20)$ of patients with compensated cirrhosis and CKD stages 4 or 5 developed serious AEs, none were reported as G/P-related. 60 No matter whether concurrent with end-stage renal disease or not, DAA-related serious AEs and AEs leading to G/P discontinuation were rare $(<1 \%) .60$ Hepatic decompensation and death occurred in 1 and 7 patients, respectively, but none were considered related to G/P. Patients with laboratory abnormalities grade $\geq 3$ were rare among both patients with compensated cirrhosis and without cirrhosis. 60 Alanine aminotransferase elevations greater than 5 times the upper limit of normal occurred in two $(<0.1 \%)$ patients without cirrhosis, and elevations of total bilirubin at least 3-times the upper limit of normal occurred in nine $(0.4 \%)$ patients, including three $(0.1 \%)$ with cirrhosis and six $(0.3 \%)$ without cirrhosis. Overall, this combined analysis demonstrated that the recommended dose of $\mathrm{G} / \mathrm{P}$ for patients with compensated liver disease and/or with any degree of renal impairment is safe and well-tolerated. The fixed-dose of $G / P$ was also demonstrated safe and tolerable in patients with psychiatric disorders, ${ }^{52}$ those with recent drug use, ${ }^{56}$ those receiving OST therapy ${ }^{57}$ or acid-reducing agents, ${ }^{58}$ and patients aged 65 years or older ${ }^{61}$ by data from clinical trials and additional subgroup analysis. The safety profile of $G / P$ in adolescents was consistent with that in adults, as demonstrated in the DORA part 1 trial published recently. ${ }^{34}$

Data from real-world studies have demonstrated that the safety is similar among clinic trials. $16,27,35-37,59$ The prevalence rate of patients with AEs was $17.7 \%(1,271 / 7,199)$, and the most commonly reported AEs were the same as in the clinical trials. Only $1 \%(55 / 5,522)$ of patients reported serious AEs, and $0.6 \%(33 / 5,595)$ of patients discontinued study treatment because of AEs. Only 4 of $2,333(0.2 \%)$ patients experienced hepatic decompensation events. 49

Although patients with $\mathrm{G} / \mathrm{P}$ had a high prevalence of AEs, ENDURANCE-2 reported that the frequency and severity of AEs and laboratory abnormalities in the HCV genotype 2-infected patients treated with G/P were similar to those treated with placebo. 38

\section{Conclusions}

Although the data from clinical trials and real-world studies described above demonstrated that $G / P$ could help a vast majority of chronic hepatitis c patients to safely eliminate $\mathrm{HCV}$, even in patients who are not fully adherent to G/P regimen, ${ }^{62}$ there are still some problems that deserve attention.

Several studies have demonstrated that the A30K substitution significantly decreases the SVR12 rate in genotype 3 patients treated with the G/P regimen. 39,63 Patients with subtype $3 \mathrm{~b}$ naturally possess $\mathrm{A} 30 \mathrm{~K}$, and over $95 \%$ of patients with subtype $3 \mathrm{~b}$ harbor the paired $\mathrm{A} 30 \mathrm{~K}+\mathrm{L} 31 \mathrm{M}$ substitutions, ${ }^{64,65}$ which showed a $>20$-fold increase in $50 \%$ effect concentration for pibrentasvir. ${ }^{64}$ Research conducted by Nozaki et al. ${ }^{22}$ and Tamori et al. ${ }^{23}$ showed SVR rates were $33.3 \%(2 / 6)$ and $50 \%(2 / 4)$, respectively. Therefore, the efficacy of G/P in patients with subtype $3 b$ is worthy of attention, especially in Asia, where the prevalence of subtype $3 b$ is much higher than that in other continents. ${ }^{66,67}$ Nevertheless, most clinical studies have been conducted in Europe and North America, where the proportion of subtype $3 \mathrm{~b}$ is less than $1 \%$ for genotype $3 .{ }^{67}$ Evaluation of the efficacy of G/P in patients with HCV subtype 3b infection is still lacking and needs further study.

All patients with NS5A-P32del have experienced virologic failure after receiving G/P treatment, according to the limited data. $23,33,40,54$ P32del confers an $>1,000$-fold change resistance to pibrentasvir and $>10,000$-fold change resistance to velpatasvir in the HCV genotype 1 b Con replicon ${ }^{54}$ appearing in $5 \%$ to $10 \%$ of genotype $1 \mathrm{~b}$ patients who experienced virologic failure with daclatasvir-containing therapies and sofosbuvir/ledipasvir treatment, but which has not been found among treatment-naïve patients. ${ }^{68,69}$ Therefore, patients with prior NS5A inhibitor treatment experience, especially those with genotype $1 \mathrm{~b}$ infection, should pay attention to P32del and switch to other treatment options when P32del occurs.

Currently, although G/P has been approved for children aged 12 years and older or weighing at least $45 \mathrm{~kg}$ without dose changing, the efficacy and safety of G/P for adolescents with genotypes 5 and 6 infections or previous sofosbuvir experience were inferred from adult data and have not been directly evaluated. ${ }^{34}$ More evidence from clinical trials and real-world studies is needed to prove effectiveness and safety in adolescents.

In conclusion, G/P is highly efficacious for and well-tolerated in chronic HCV-infected patients with compensated liver disease, including patients with compensated cirrhosis, $\mathrm{HCV} /$ human immunodeficiency virus coinfection, end-stage renal disease, liver or kidney transplants, recent drug use or in adolescents, according to data from clinic trails and real-world studies. In the DAA era, the characteristics of HCV patients have changed greatly, including for treatmentexperienced patients and patients with cirrhosis that has decreased over time. ${ }^{70,71}$ The fixed-dose G/P regimen for 8-week duration has been approved for treatment-naïve $\mathrm{HCV}$-infected patients with compensated liver disease (with or without cirrhosis) by the European Commission ${ }^{72}$ and the USA Food and Drug Administration, ${ }^{8}$ which means a shorter duration of therapy can benefit the vast majority of chronic hepatitis c patients. However, patients with HCV subtype $3 \mathrm{~b}$ infection or NS5A-P32del need special attention.

\section{Funding}

This work was supported in part by the Kuanren Talents Program of the Second Affiliated Hospital of Chongqing Medical University.

\section{Conflict of interest}

The authors have no conflict of interests related to this publication. 
Liu X. et al: G/P effectiveness and tolerability

\section{Author contributions}

Wrote the manuscript $(\mathrm{XL})$, critically revised the manuscript and provided study supervision $(\mathrm{PH})$.

\section{References}

[1] World Health Organization. Global hepatitis report, 2017. Available from: https://apps.who.int/iris/bitstream/handle/10665/255016/9789241565455eng.pdf.

[2] Ioannou GN, Feld JJ. What are the benefits of a sustained virologic response to direct-acting antiviral therapy for hepatitis C virus infection? Gastroenterology 2019;156:446-460.e2. doi:10.1053/j.gastro.2018.10.033.

[3] Asselah T, Marcellin P, Schinazi RF. Treatment of hepatitis C virus infection with direct-acting antiviral agents: $100 \%$ cure? Liver Int 2018;38(Supp 1):7-13. doi:10.1111/liv.13673

[4] Foster GR, Afdhal N, Roberts SK, Bräu N, Gane EJ, Pianko S, et al. Sofosbuvir and velpatasvir for HCV genotype 2 and 3 infection. $N$ Engl J Med 2015;373:2608-2617. doi:10.1056/NEJMoa1512612.

[5] Jacobson IM, Lawitz E, Gane EJ, Willems BE, Ruane PJ, Nahass RG, et al. Efficacy of 8 weeks of sofosbuvir, velpatasvir, and voxilaprevir in patients with chronic HCV infection: 2 phase 3 randomized trials. Gastroenterology 2017;153:113-122. doi:10.1053/j.gastro.2017.03.047

[6] Hezode C, Reau N, Svarovskaia ES, Doehle BP, Shanmugam R, DvorySobol $\mathrm{H}$, et al. Resistance analysis in patients with genotype 1-6 HCV infection treated with sofosbuvir/velpatasvir in the phase III studies. J Hepato 2018;68:895-903. doi:10.1016/j.jhep.2017.11.032.

[7] Yao BB, Fredrick LM, Schnell G, Kowdley KV, Kwo PY, Poordad F, et al. Efficacy and safety of glecaprevir/pibrentasvir in patients with HCV genotype 5/6: An integrated analysis of phase $2 / 3$ studies. Liver Int 2020;40:23855/6: An integrated analysis of
2393. doi: $10.1111 /$ liv.14535.

[8] MAVYRET@(glecaprevir and pibrentasvir), US prescribing information. Available from: https://www.rxabbvie.com/pdf/mavyret_pi.pdf.

[9] Sorbo MC, Cento V, Di Maio VC, Howe AYM, Garcia F, Perno CF, et al. Hepatitis $C$ virus drug resistance associated substitutions and their clinical relevance: Update 2018. Drug Resist Updat 2018;37:17-39. doi:10.1016/j. drup.2018.01.004.

[10] Suleiman AA, Lin CW, Liu W, Eckert D, Mensing S, Burroughs M, et al. Population pharmacokinetics of glecaprevir/pibrentasvir in HCV-infected Japanese subjects in phase 3 CERTAIN-1 and CERTAIN-2 trials. J Clin Pharmacol 2020;60:331-339. doi:10.1002/jcph.1524.

[11] Lin CW, Dutta S, Ding B, Wang T, Zadeikis N, Asatryan A, et al. Pharmacokinetics, safety, and tolerability of glecaprevir and pibrentasvir in healthy white, Chinese, and Japanese adult subjects. J Clin Pharmacol 2017;57: 1616-1624. doi: $10.1002 /$ jcph.959.

[12] Smolders EJ, Berden FA, de Kanter CT, Kievit W, Drenth JP, Burger DM. The majority of hepatitis $C$ patients treated with direct acting antivirals are at risk for relevant drug-drug interactions. United European Gastroenterol 2017;5:648-657. doi:10.1177/2050640616678151.

[13] Chayama $K$, Suzuki $F$, Karino $Y$, Kawakami $Y$, Sato $K$, Atarashi $T$, et al. Efficacy and safety of glecaprevir/pibrentasvir in Japanese patients with chronic genotype 1 hepatitis $C$ virus infection with and without cirrhosis. J chronic genotype 1 hepatitis C virus infection with and without cirrhosi
Gastroenterol 2018;53:557-565. doi:10.1007/s00535-017-1391-5.

[14] Toyoda H, Chayama K, Suzuki F, Sato K, Atarashi T, Watanabe T, et al. Efficacy and safety of glecaprevir/pibrentasvir in Japanese patients with chronic genotype 2 hepatitis C virus infection. Hepatology 2018;67:505513. doi:10.1002/hep.29510.

[15] Asselah T, Lee SS, Yao BB, Nguyen T, Wong F, Mahomed A, et al. Efficacy and safety of glecaprevir/pibrentasvir in patients with chronic hepatitis $C$ virus genotype 5 or 6 infection (ENDURANCE-5,6): an open-label, multicentre, phase 3b trial. Lancet Gastroenterol Hepatol 2019;4:45-51. doi:10.1016/S2468-1253(18)30341-8.

[16] Berg T, Naumann U, Stoehr A, Sick C, John C, Teuber G, et al. Real-world effectiveness and safety of glecaprevir/pibrentasvir for the treatment of chronic hepatitis C infection: data from the German Hepatitis C-Registry. Aliment Pharmacol Ther 2019;49:1052-1059. doi:10.1111/apt.15222.

[17] Brown RS Jr, Buti M, Rodrigues L, Chulanov V, Chuang WL, Aguilar $\mathrm{H}$, et al. Glecaprevir/pibrentasvir for 8 weeks in treatment-naïve patients with chronic HCV genotypes 1-6 and compensated cirrhosis: The EXPEDITION-8 trial. J Hepatol 2020;72:441-449. doi:10.1016/j.jhep.2019.10.020.

[18] Forns X, Lee SS, Valdes ], Lens S, Ghalib R, Aguilar H, et al. Glecaprevir plus pibrentasvir for chronic hepatitis $C$ virus genotype 1, 2, 4, 5, or 6 infection in adults with compensated cirrhosis (EXPEDITION-1): a single-arm, openlabel, multicentre phase 3 trial. Lancet Infect Dis 2017;17:1062-1068. doi:10.1016/S1473-3099(17)30496-6

[19] Kwo PY, Poordad F, Asatryan A, Wang S, Wyles DL, Hassanein T, et al. Glecaprevir and pibrentasvir yield high response rates in patients with HCV genotype 1-6 without cirrhosis. J Hepatol 2017;67:263-271. doi:10.1016/ j.jhep.2017.03.039.

[20] Wyles D, Poordad F, Wang S, Alric L, Felizarta F, Kwo PY, et al. Glecaprevir/ pibrentasvir for hepatitis C virus genotype 3 patients with cirrhosis and/ or prior treatment experience: A partially randomized phase 3 clinical trial. Hepatology 2018;67:514-523. doi:10.1002/hep.29541.

[21] Soria A, Fava M, Bernasconi DP, Lapadula G, Colella E, Valsecchi MG, et al. Comparison of three therapeutic regimens for genotype- 3 hepatitis $C$ virus infection in a large real-life multicentre cohort. Liver Int 2020;40:769-777. doi:10.1111/liv.14386.
[22] Nozaki A, Atsukawa M, Kondo C, Toyoda H, Chuma M, Nakamuta M, et al. The effectiveness and safety of glecaprevir/pibrentasvir in chronic hepatitis $C$ patients with refractory factors in the real world: a comprehensive analysis of a prospective multicenter study. Hepatol Int 2020;14:225-238. doi: 10.1007/s12072-020-10019-z.

[23] Tamori A, Inoue K, Kagawa T, Takaguchi K, Nouso K, Iwasaki Y, et al. Intention-to-treat assessment of glecaprevir + pibrentasvir combination therapy for patients with chronic hepatitis $\mathrm{C}$ in the real world. Hepatol Res 2019;49:1365-1373. doi:10.1111/hepr.13410.

[24] Toyoda H, Atsukawa M, Watanabe T, Nakamuta M, Uojima H, Nozaki A, et al. Real-world experience of 12 -week direct-acting antiviral regimen of glecaprevir and pibrentasvir in patients with chronic hepatitis $C$ virus infection. J Gastroenterol Hepatol 2020;35:855-861. doi:10.1111/jgh.14874.

[25] Gane E, Lawitz E, Pugatch D, Papatheodoridis G, Bräu N, Brown A, et al. Glecaprevir and pibrentasvir in patients with HCV and severe renal impairment. N Engl J Med 2017;377:1448-1455. doi:10.1056/NEJMoa1704053.

[26] Lawitz E, Flisiak R, Abunimeh M, Sise ME, Park JY, Kaskas M, et al. Efficacy and safety of glecaprevir/pibrentasvir in renally impaired patients
with chronic HCV infection. Liver Int 2020;40:1032-1041. doi:10.1111/ with chron.

[27] Atsukawa M, Tsubota A, Toyoda H, Takaguchi K, Nakamuta M, Watanabe $\mathrm{T}$, et al. The efficacy and safety of glecaprevir plus pibrentasvir in 141 patients with severe renal impairment: a prospective, multicenter study. Aliment Pharmacol Ther 2019;49:1230-1241. doi:10.1111/apt.15218.

[28] Liu CH, Yang SS, Peng CY, Lin WT, Liu CJ, Su TH, et al. Glecaprevir/pibrentasvir for patients with chronic hepatitis $\mathrm{C}$ virus infection and severe renal impairment. J Viral Hepat 2020;27:568-575. doi:10.1111/jvh.13265.

[29] Rockstroh JK, Lacombe K, Viani RM, Orkin C, Wyles D, Luetkemeyer AF, et al. Efficacy and safety of glecaprevir/pibrentasvir in patients coinfected with hepatitis $C$ virus and human immunodeficiency virus type 1: The EXPEDITION-2 study. Clin Infect Dis 2018;67:1010-1017. doi:10.1093/cid/ ciy 220.

[30] Reau N, Kwo PY, Rhee S, Brown RS Jr, Agarwal K, Angus P, et al. Glecaprevir/pibrentasvir treatment in liver or kidney transplant patients with hepatitis C virus infection. Hepatology 2018;68:1298-1307. doi:10.1002/ hep. 30046 .

[31] Ueda Y, Kobayashi T, Ikegami T, Miuma S, Mizuno S, Akamatsu N, et al. Efficacy and safety of glecaprevir and pibrentasvir treatment for 8 or 12 weeks in patients with recurrent hepatitis $C$ after liver transplantation: a Japanese multicenter experience. J Gastroenterol 2019;54:660-666. doi:10.1007/ s00535-019-01561-1.

[32] Poordad F, Pol S, Asatryan A, Buti M, Shaw D, Hézode C, et al. Glecaprevir/ Pibrentasvir in patients with hepatitis $C$ virus genotype 1 or 4 and past direct-acting antiviral treatment failure. Hepatology 2018;67:1253-1260. doi:10.1002/hep. 29671.

[33] Uemura H, Uchida Y, Kouyama JI, Naiki K, Tsuji S, Sugawara K, et al. NS5AP32 deletion as a factor involved in virologic failure in patients receiving glecaprevir and pibrentasvir. J Gastroenterol 2019;54:459-470. doi:10.1007/ s00535-018-01543-9.

[34] Jonas MM, Squires RH, Rhee SM, Lin CW, Bessho K, Feiterna-Sperling C, et al. Pharmacokinetics, safety, and efficacy of glecaprevir/pibrentasvir in adolescents with chronic hepatitis $C$ virus: Part 1 of the DORA study. Hepatology 2020;71:456-462. doi:10.1002/hep.30840.

[35] D'Ambrosio R, Pasulo L, Puoti M, Vinci M, Schiavini M, Lazzaroni S, et al. Real-world effectiveness and safety of glecaprevir/pibrentasvir in $723 \mathrm{pa}$ tients with chronic hepatitis C. J Hepatol. 2019;70:379-387. doi:10.1016/j. jhep.2018.11.011.

[36] Hsu SJ, Chiu MC, Fang YJ, Yang TH, Yu JJ, Chen CC, et al. Real-world effectiveness and safety of glecaprevir/pibrentasvir in Asian patients with chronic hepatitis C. J Formos Med Assoc. 2019;118:1187-1192. doi:10.1016/ j.jfma.2019.06.014.

[37] Ikeda H, Watanabe T, Atsukawa M, Toyoda H, Takaguchi K, Nakamuta M, et al. Evaluation of 8-week glecaprevir/pibrentasvir treatment in direct-acting antiviral-naïve noncirrhotic HCV genotype 1 and 2infected patients in a real-world setting in Japan. J Viral Hepat 2019;26:1266-1275. doi:10.1111/ jvh. 13170 .

[38] Asselah T, Kowdley KV, Zadeikis N, Wang S, Hassanein T, Horsmans Y, et al. Efficacy of glecaprevir/pibrentasvir for 8 or 12 weeks in patients with hepatitis C virus genotype 2, 4, 5, or 6 infection without cirrhosis. Clin Gastroenterol Hepatol 2018;16:417-426. doi:10.1016/j.cgh.2017.09.027.

[39] Zeuzem S, Foster GR, Wang S, Asatryan A, Gane E, Feld JJ, et al. Glecaprevir-pibrentasvir for 8 or 12 weeks in HCV genotype 1 or 3 infection. N Engl J Med 2018;378:354-369. doi:10.1056/NEJMoa1702417.

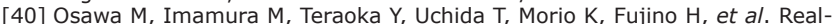
world efficacy of glecaprevir plus pibrentasvir for chronic hepatitis $\mathrm{C}$ patient with previous direct-acting antiviral therapy failures. J Gastroenterol tient with previous direct-acting antiviral therapy failure

[41] Fontana RJ, Lens S, McPherson S, Elkhashab M, Ankoma-Sey V, Bondin M, et al. Efficacy and safety of 8 weeks of glecaprevir/pibrentasvir in treatmentnaïve, HCV-infected patients with APRI $\leq 1$ in a single-arm, open-label, multicenter study. Adv Ther 2019;36:3458-3470. doi:10.1007/s12325-01901123-0.

[42] Lok AS, Sulkowski MS, Kort J], Willner I, Reddy KR, Shiffman ML, et al. Efficacy of glecaprevir and pibrentasvir in patients with genotype 1 hepatitis $C$ virus infection with treatment failure after NS5A inhibitor plus sofosbuvir therapy. Gastroenterology 2019;157:1506-1517.e1. doi:10.1053/j.gastro. 2019.08.008.

[43] Persico M, Aglitti A, Milella M, Coppola C, Messina V, Claar E, et al. Real-life glecaprevir/pibrentasvir in a large cohort of patients with hepatitis $C$ virus infection: The MISTRAL study. Liver Int 2019;39:1852-1859. doi:10.1111/ liv. 14170. 
[44] Liu CH, Liu CJ, Hung CC, Hsieh SM, Su TH, Sun HY, et al. Glecaprevir/pibrentasvir for patients with chronic hepatitis $C$ virus infection: Real-world effectiveness and safety in Taiwan. Liver Int 2020;40:758-768. doi:10.1111/ liv. 14295.

[45] Sugiura A, Joshita S, Yamashita Y, Yamazaki T, Fujimori N, Kimura T, et al. Effectiveness of glecaprevir/pibrentasvir for hepatitis C: Real-world experience and clinical features of retreatment cases. Biomedicines 2020;8:74. doi: $10.3390 /$ biomedicines 8040074

[46] Kusakabe A, Kurosaki M, Itakura J, Joko K, Akahane T, Tsuji K, et al. Efficacy and safety of glecaprevir/pibrentasvir as retreatment therapy for patients with genotype 2 chronic hepatitis $C$ who failed prior sofosbuvir plus ribavirin regimen. Hepatol Res 2019;49:1121-1126. doi:10.1111/hepr. 13387.

[47] Sezaki H, Suzuki F, Hosaka T, Fujiyama S, Kawamura Y, Akuta N, et al. Initial- and re-treatment effectiveness of glecaprevir and pibrentasvir for Japanese patients with chronic hepatitis $C$ virus-genotype $1 / 2 / 3$ infections. J Gastroenterol 2019;54:916-927. doi:10.1007/s00535-019-01575-9.

[48] Puoti M, Foster GR, Wang S, Mutimer D, Gane E, Moreno C, et al. High SVR12 with 8-week and 12-week glecaprevir/pibrentasvir therapy: An integrated analysis of HCV genotype 1-6 patients without cirrhosis. J Hepatol 2018;69:293-300. doi:10.1016/j.jhep.2018.03.007.

[49] Lampertico P, Carrión JA, Curry M, Turnes J, Cornberg M, Negro F, et al. Real-world effectiveness and safety of glecaprevir/pibrentasvir for the treatment of patients with chronic HCV infection: A meta-analysis. J Hepatol 2020;72:1112-1121. doi:10.1016/j.jhep.2020.01.025.

[50] Flamm S, Mutimer D, Asatryan A, Wang S, Rockstroh J, Horsmans Y, et al. Glecaprevir/Pibrentasvir in patients with chronic HCV genotype 3 infecGlecaprevir/Pibrentasvir in patients with chronic HCV genotype 3 infec-
tion: An integrated phase 2/3 analysis. J Viral Hepat 2019;26:337-349. tion: An integrated phase
doi: $10.1111 /$ jvh.13038.

[51] Morishita A, Ogawa C, Moriya A, Tani J, Yoneyama H, Fujita K, et al. Clinical outcomes of hepatitis $C$ virus elimination using glecaprevir and pibrentasvir in hemodialysis patients: A multicenter study. Hepatol Res 2020;50: 557-564. doi:10.1111/hepr.13482.

[52] Back D, Belperio P, Bondin M, Negro F, Talal AH, Park C, et al. Efficacy and safety of glecaprevir/pibrentasvir in patients with chronic HCV infection and psychiatric disorders: An integrated analysis. J Viral Hepat 2019;26: 951-960. doi:10.1111/jvh.13110.

[53] Poordad F, Felizarta F, Asatryan A, Sulkowski MS, Reindollar RW, Landis CS, et al. Glecaprevir and pibrentasvir for 12 weeks for hepatitis C virus genotype 1 infection and prior direct-acting antiviral treatment. Hepatology 2017;66:389-397. doi:10.1002/hep.29081.

[54] Krishnan P, Schnell G, Tripathi R, Beyer J, Reisch T, Dekhtyar T, et al. Integrated resistance analysis of CERTAIN-1 and CERTAIN-2 studies in hepatitis $C$ virus-infected patients receiving glecaprevir and pibrentasvir in Japan. Antimicrob Agents Chemother 2018;62:e02217-17. doi:10.1128/ AAC. 02217-17.

[55] Degenhardt L, Peacock A, Colledge S, Leung J, Grebely J, Vickerman P, et al. Global prevalence of injecting drug use and sociodemographic characteristics and prevalence of HIV, HBV, and HCV in people who inject drugs: a multistage systematic review. Lancet Glob Health 2017;5:e1192-e1207. doi:10.1016/S2214-109X(17)30375-3

[56] Foster GR, Dore GJ, Wang S, Grebely J, Sherman KE, Baumgarten A, et al. Glecaprevir/pibrentasvir in patients with chronic HCV and recent drug use: An integrated analysis of 7 phase III studies. Drug Alcohol Depend 2019;194:487-494. doi:10.1016/j.drugalcdep.2018.11.007.

[57] Grebely J, Dore GJ, Alami NN, Conway B, Dillon JF, Gschwantler M, et al. Safety and efficacy of glecaprevir/pibrentasvir in patients with chronic hepatitis $C$ genotypes 1-6 receiving opioid substitution therapy. Int J Drug Policy 2019;66:73-79. doi:10.1016/j.drugpo.2019.01.011.

[58] Flamm S, Reddy KR, Zadeikis N, Hassanein T, Bacon BR, Maieron A, et al. Efficacy and pharmacokinetics of glecaprevir and pibrentasvir with con- current use of acid-reducing agents in patients with chronic HCV infection. Clin Gastroenterol Hepatol 2019;17:527-535.e6. doi:10.1016/j.cgh. 2018.07.003.

[59] Kiser J]. Safety of hepatitis C viral protease inhibitors in compensated cirrhotics: Lingering concerns put to rest? Clin Infect Dis 2019;69:16651666. doi: $10.1093 /$ cid/ciz025.

[60] Gane E, Poordad F, Zadeikis N, Valdes J, Lin CW, Liu W, et al. Safety and pharmacokinetics of glecaprevir/pibrentasvir in adults with chronic genotype 1-6 hepatitis $C$ virus infections and compensated liver disease. Clin type 1-6 hepatitis C virus infections and compensated

[61] Foster GR, Asselah T, Kopecky-Bromberg S, Lei Y, Asatryan A, Trinh R, et al. Safety and efficacy of glecaprevir/pibrentasvir for the treatment of chronic hepatitis $C$ in patients aged 65 years or older. PLoS One 2019; 14:e0208506. doi:10.1371/journal.pone.0208506.

[62] Brown A, Welzel TM, Conway B, Negro F, Bräu N, Grebely J, et al. Adherence to pan-genotypic glecaprevir/pibrentasvir and efficacy in HCV-infected patients: A pooled analysis of clinical trials. Liver Int 2020;40:778-786. doi:10.1111/liv. 14266 .

[63] Singh AD, Maitra S, Singh N, Tyagi P, Ashraf A, Kumar R, et al. Systematic review with meta-analysis: impact of baseline resistance-associated substitutions on the efficacy of glecaprevir/pibrentasvir among chronic hepatitis C patients. Aliment Pharmacol Ther 2020;51:490-504. doi:10.1111/ apt.15633.

[64] Smith D, Magri A, Bonsall D, Ip CLC, Trebes A, Brown A, et al. Resistance analysis of genotype 3 hepatitis $C$ virus indicates subtypes inherently resistant to nonstructural protein 5A inhibitors. Hepatology 2019;69:18611872. doi: 10.1002/hep.29837.

[65] Wei L, Omata M, Lim YS, Xie Q, Hou JL, Jia J, et al. HCV phylogenetic signature and prevalence of pretreatment NS5A and NS5B NI-Resistance associated substitutions in HCV-Infected patients in Mainland China. Antiviral Res 2018;158:178-184. doi:10.1016/j.antiviral.2018.08.001.

[66] Chen Y, Yu C, Yin X, Guo X, Wu S, Hou J. Hepatitis C virus genotypes and subtypes circulating in Mainland China. Emerg Microbes Infect 2017;6:e95. doi:10.1038/emi.2017.77.

[67] Welzel TM, Bhardwaj N, Hedskog C, Chodavarapu K, Camus G, McNally J, et al. Global epidemiology of HCV subtypes and resistance-associated substitutions evaluated by sequencing-based subtype analyses. J Hepatol 2017;67:224-236. doi:10.1016/j.jhep.2017.03.014.

[68] Sato K, Uraoka T. Challenge to overcome: Nonstructural protein 5A-P32 deletion in direct-acting antiviral-based therapy for hepatitis $C$ virus. World J Gastroenterol 2018;24:4304-4310. doi:10.3748/wjg.v24.i38.4304.

[69] Hikita H, Takehara T. NS5A-P32 deletion in hepatitis C genotype $1 \mathrm{~b}$ infection is the most refractory treatment-mediated amino acid change exhibiting resistance to all NS5A inhibitors. Semin Liver Dis 2020;40:143-153. do i: $10.1055 / \mathrm{s}-0039-3402001$.

[70] Tsai N, Bacon B, Curry M, Flamm SL, Milligan S, Wick N, et al. Changing demographics among populations prescribed HCV treatment, 2013-2017. demographics among populations presc

[71] Christensen S, Ingiliz P, Schulze Zur Wiesch J, Schewe K, Boesecke C, Hüppe D, et al. Significant changes of HCV patient characteristics over time in the era of direct antiviral agent therapy - are all HCV subpopulations treated similarly? - Results from the German hepatitis C Cohort. J Hepatol 2018;68:S289-S290. doi:10.1016/S0168-8278(18)30798-0.

[72] European Commission Grants AbbVie Marketing Authorization Shortening MAVIRET $®$ (glecaprevir/pibrentasvir) treatment duration to eight weeks for treatment-naïve chronic HCV patients with genotype 3 and compensated cirrhosis. Available from: https://news.abbvie.com/news/press-releases/ cirrhosis. Available from: https://news.abbvie.com/news/press-releases/ european-commission-grants-abbvie-marketing-authorization-shortening-
maviret-glecaprevirpibrentasvir-treatment-duration-to-eight-weeks-fortreatment-nave-chronic-hcv-patients-with-genotype-3-and-compensatedcirrhosis.htm?view_id=4284. 